\title{
Avaliação de diferentes crioprotetores intra e extracelulares na criopreservação de sêmen de touros
}

\author{
[Evaluation of different intra and extracellular cryoprotectants on bull semen cryopreservation] \\ E.M. Madeira ${ }^{1}$, I. Bianchi ${ }^{1}$, M.B. Vieira ${ }^{2}$, A. Schneider ${ }^{1}$, N.C. Severo ${ }^{2}$, \\ L.F.M. Pfeifer ${ }^{3}$, M.N. Corrêa $a^{1}$ \\ ${ }^{1}$ Faculdade de Veterinária - Universidade Federal de Pelotas - Pelotas, RS \\ ${ }^{2}$ Médico veterinário autônomo \\ ${ }^{3}$ Embrapa Rondônia
}

\begin{abstract}
RESUMO
Em um delineamento experimental usando o fatorial 3x2, três crioprotetores internos, glicerol (GLI), etilenoglicol (EG) e dimetilformamida (DMF), e dois externos, gema de ovo (GEMA) e lipoproteína de baixa densidade (LDL), avaliaram-se a motilidade ao descongelamento de GLI-GEMA 53,9 $\pm 1,96$, sendo superior aos demais tratamentos $(\mathrm{P}<0,05)$. Na avaliação de morfologia ao descongelamento, não houve diferença $(\mathrm{P}>0,05)$ entre os tratamentos EG-GEMA 68,3 $\pm 1,58$, EG-LDL 72,2 $\pm 2,39$ e DMF-GEMA $68,7 \pm 1,67$ que foram mais altos que os demais $(\mathrm{P}<0,05)$. A avaliação de integridade de membrana por

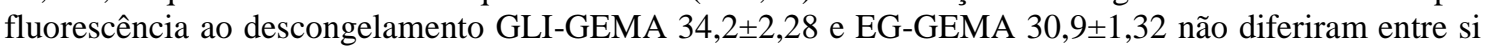
$(\mathrm{P}>0,05)$, mas foram mais elevados que os demais $(\mathrm{P}<0,05)$, enquanto que a HOST dos tratamentos DMF-GEMA 13,6 $\pm 1,30$ e DMF-LDL 9,8 $\pm 0,78$ diferirem entre si $(\mathrm{P}<0,05)$ e foram mais baixas que as demais $(\mathrm{P}<0,05)$. $\mathrm{O}$ uso de etilenoglicol associado à gema de ovo pode ser uma alternativa ao uso de glicerol nos protocolos de congelamento de sêmen de touros.
\end{abstract}

Palavras-chave: bovino, sêmen, glicerol, etilenoglicol, dimetilformamida, LDL

\begin{abstract}
The experiment was designed as $3 \times 2$ factorial design, with three internal cryoprotectants, glycerol $(G L Y)$, etileneglycol (EG) and dymethilformamide (DMF) and two external, egg yolk (YOLK) and density low lipoproteina $(L D L)$. The motility at thawing for GLY-YOLK (53.9 \pm 1.96$)$ was higher than other treatments $(P<0.05)$. The percentage of cells with normal morphology at thawing was not different

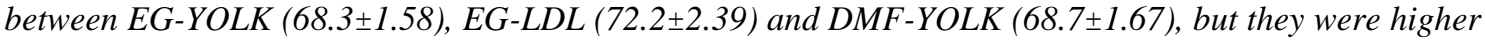
than the others $(P<0.05)$. The evaluation of membrane integrity through fluorescent probes at thawing

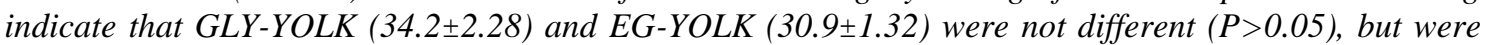
higher than the others $(P<0.05)$. The evaluation of membrane integrity through hypoosmotic swelling test (HOST) indicate that DMF-YOLK (13.6 \pm 1.30$)$ and DMF-LDL $(9.8 \pm 0.78)$ were different $(P<0.05)$ and lower than the others $(P<0.05)$. The use of ethylene glycol associated to egg yolk can be a viable alternative to the use of glycerol in bull semen freezing protocols.
\end{abstract}

Keywords: bull, sperm, glycerol, ethylene glycol, dimethylformamide, LDL

\section{INTRODUÇÃO}

$\mathrm{Na}$ criopreservação da célula espermática, os danos sofridos durante o processo de preservação é um dos principais assuntos estudados, por Alvarenga et al. (2000), Watson (2000) e Ball e
Vo (2001). Alternativas ao uso do glicerol em protocolos de congelamento de sêmen têm sido pesquisadas na maioria das espécies. Nesse contexto, o etilenoglicol tem sido usado com sucesso em diluentes para sêmen equino e em pequena escala para sêmen bovino, apresentando

Recebido em 1 de outubro de 2011

Aceito em 1 de outubro de 2012

E-mail: elisangelamadeira@yahoo.com.br 
vantagens frente ao glicerol e ao dimetilsulfoxido, como foi observado por Guthrie et al. (2002). As amidas também já foram utilizadas na criopreservação de sêmen bovino por Nagase et al. (1972). Recentemente as amidas foram adicionadas com bastante êxito como crioprotetores em meios de preservação de sêmen equino (Medeiros et al., 2002; Squires et al., 2004) e também de suínos (Bianchi et al., 2008), galos (Tselutin et al., 1999) e peixes (Ogier de Baulny et al., 1999).

Tradicionalmente o crioprotetor extracelular utilizado para sêmen bovino é a gema de ovo, cuja função crioprotetora é exercida pela lipoproteína de baixa densidade (LDL) presente na gema. A LDL purificada já foi utilizada como crioprotetor externo em diluentes para sêmen de touros (Amirat et al., 2004), suínos (Jiang et al., 2007) e cães (Varela Junior et al., 2009). A LDL interage com as proteínas do plasma seminal, prevenindo a interação destas com a membrana do espermatozoide (Bergeron e Manjunath, 2006); além disso, ocorre o influxo dos fosfolipídios e colesterol da membrana espermática com uma melhor resistência ao choque do congelamento (Manjunath e Thérien, 2002; Bergeron et al., 2004).

Diante do exposto, o presente trabalho teve como objetivo avaliar o efeito da criopreservação do sêmen de touros utilizando dois crioprotetores externos (gema de ovo e a LDL) e três internos (glicerol, etilenoglicol e dimetilformamida).

\section{MATERIAL E MÉTODOS}

O sêmen utilizado foi proveniente de uma central de coleta e produção de sêmen, obtido de sete touros das raças Hereford, Aberdeen Angus, Red Angus e Red Brangus, mantidos em regime de coleta semanal. De cada reprodutor, usaram-se sete ejaculados para avaliar a motilidade e o vigor espermático por microscopia ótica em aumento de 200x. Somente ejaculados com motilidade $\geq 70 \%$ e vigor $\geq 3$, de uma escala de 0 a 5, foram utilizados para congelamento, conforme recomendação do Manual de coleta e processamento de sêmen (CBRA, 1998). A concentração espermática foi obtida por meio de espectrofotometria (Accucell photometer ${ }^{\circledR}$, IMV, France), sendo o sêmen envasado em palhetas médias de 0,5ml (IMV, France), a fim de atingir a concentração de $30 \times 10^{6}$ espermatozoides por palheta pré-congelamento. A composição básica dos diluentes utilizados continha: $5,8 \%$ de solução estoque de citrato de sódio a $29,6 \%$ e um combinado de antibióticos à base de tilosina, gentamicina, lincomicina e espectinomicina (Shin et al., 1988).

Seis diluentes foram testados utilizando os crioprotetores intracelulares glicerol (G 4094, Merck; GLI), dimetilformamida (D4254, Sigma; DMF) e etilenoglicol (324558, Sigma; EG) combinados com os crioprotetores extracelulares gema de ovo (GEMA) e lipoproteína de baixa densidade (LDL). As combinações e concentrações dos crioprotetores utilizados foram: GLI 7\% + GEMA 20\% (GLI-GEMA); DMF 5\% + GEMA 20\% (DMF-GEMA); EG 7\% + GEMA 20\% (EG-GEMA); GLI 7\% + LDL $8 \%$ (GLI-LDL); DMF 5\% + LDL 8\% (DMF-LDL); EG $7 \%+$ LDL 8\% (EG-LDL). Para cada tratamento foram congeladas 10 palhetas.

O método usado para extração do LDL foi o descrito por Moussa et al. (2002), com o qual se obtém pureza de $97 \%$. Após o envase, o sêmen foi submetido à curva de resfriamento a $10^{\circ} \mathrm{C}$ por $60 \mathrm{~min}$ e posteriormente a $5^{\circ} \mathrm{C}$ por mais $60 \mathrm{~min}$, seguida de congelamento em sistema automático de congelamento e armazenamento em nitrogênio líquido $\left(-196^{\circ} \mathrm{C}\right)$.

O descongelamento das palhetas foi realizado em banho-maria a $37^{\circ} \mathrm{C}$ durante 30 s. A avaliação da motilidade espermática foi realizada quando a curva de congelamento atingiu $5^{\circ} \mathrm{C}$ e após o descongelamento das palhetas, mediante microscopia ótica em aumento de 200x (Bearden e Fuquay, 1997). Para isso, foi incubada durante $10 \mathrm{~min}$ em banho-maria a $37^{\circ} \mathrm{C}$. Na avaliação da morfologia espermática após o descongelamento, mediante avaliação de 200 células, utilizou-se microscópio de contraste de fases (CBRA, 1998). Após o descongelamento também foi avaliada a integridade de membrana plasmática, por meio de fluorescência e de choque hiposmótico (HOST). A avaliação de integridade da membrana plasmática por fluorescência foi realizada por meio de sondas de diacetato de carboxifluoresceína (CFDA; C5041) e iodeto de propídio (PI; P4170) (Harrison e Vickers, 1990). Foi feita em microscópio de epifluorescência (Olympus BX 51, América Inc., Sapporo, Japan) através de excitação em filtro WU sob aumento de 400x. Foram avaliados 100 espermatozoides 
em uma mesma lâmina e classificados conforme sua coloração, em íntegros espermatozoides corados em verde em toda sua extensão e lesados espermatozoides corados em vermelho. A integridade funcional da membrana espermática avaliada pelo HOST seguiu as recomendações de Vasquez et al. (1997). A Foi feita utilizando-se câmara de Neubauer e microscopia óptica com contraste de fases em aumento de 400x, procedendo-se à leitura de 100 células e registrando 0 número de células que apresentavam cauda do espermatozoide normal em relação àquelas com cauda dobrada ou enrolada. $\mathrm{O}$ valor que foi utilizado para análise correspondeu à diferença obtida entre o número de espermatozoides com cauda dobrada e enrolada observadas no teste realizado com a solução hiposmótica $(0 \mathrm{mOsm} / \mathrm{kg})$ e isosmótica $(312 \mathrm{mOsm} / \mathrm{kg})$.

A análise estatística seguiu um delineamento fatorial $3 \times 2$, representando as combinações dos crioprotetores intracelulares GLI, DMF e EG, e extracelulares GEMA e LDL. As variáveis dependentes consideradas foram: motilidade a $5^{\circ} \mathrm{C}$ e ao descongelamento, espermatozoides com morfologia normal ao descongelamento e espermatozoides com membrana íntegra no descongelamento, no teste da fluorescência e no
HOST. Usou-se o least significant difference (LSD) para comparação de médias, empregando o procedimento GLM do SAS (Statistical..., 1999).

\section{RESULTADOS}

Os resultados das avaliações espermáticas encontram-se na Tab. 1. Na avaliação précongelamento a $5^{\circ} \mathrm{C}$, os tratamentos GLI-GEMA e EG-LDL permaneceram com motilidade acima de $70 \%$ acima dos demais tratamentos $(\mathrm{P}<0,05)$. No entanto, após o descongelamento, GLIGEMA permaneceu alto em relação aos demais $(\mathrm{P}<0,05)$, enquanto os tratamentos que incluíram o etilenoglicol, independentemente da combinação EG-GEMA ou EG-LDL, não diferiram entre si $(\mathrm{P}>0,05)$. Mesmo que a motilidade após o descongelamento seja considerada satisfatória para o tratamento GLILDL, ela foi diferente $(\mathrm{P}<0,05)$ da combinação do GLI-GEMA. Os tratamentos que incluíram DMF com GEMA ou com LDL tiveram os piores resultados de motilidade após o descongelamento, ficando abaixo da recomendação mínima de $30 \%$ sugerida pelo CBRA (1998), não sendo diferentes entre si $(\mathrm{P}>0,05)$.

Tabela 1. Características espermáticas pré e pós-descongelamento (média \pm SEM) de sêmen de touro de acordo com os tratamentos utilizados

\begin{tabular}{|c|c|c|c|c|c|c|}
\hline Parâmetros & $\begin{array}{l}\text { GLI- } \\
\text { GEMA }\end{array}$ & $\begin{array}{l}\text { DMF- } \\
\text { GEMA }\end{array}$ & $\begin{array}{l}\text { GLI- } \\
\text { LDL }\end{array}$ & $\begin{array}{l}\text { DMF- } \\
\text { LDL }\end{array}$ & $\begin{array}{c}\text { EG- } \\
\text { GEMA }\end{array}$ & $\begin{array}{l}\text { EG- } \\
\text { LDL }\end{array}$ \\
\hline Motilidade a $5^{\circ} \mathrm{C}, \%$ & $70,4 \pm 0,38 \mathrm{a}$ & $65,7 \pm 1,17 \mathrm{c}$ & $57,4 \pm 2,59 \mathrm{e}$ & $60,9 \pm 2,15 \mathrm{~d}$ & $67,5 \pm 0,63 b$ & $70,0 \pm 1,03 \mathrm{a}$ \\
\hline $\begin{array}{l}\text { Motilidade após o } \\
\text { descongelamento, \% }\end{array}$ & $53,9 \pm 1,96 a$ & $9,6 \pm 1,15 \mathrm{~d}$ & $37,6 \pm 3,36 c$ & $10,7 \pm 1,33 \mathrm{~d}$ & $45,7 \pm 2,27 \mathrm{~b}$ & $45,4 \pm 3,24 b$ \\
\hline $\begin{array}{l}\text { Morfologia normal após } \\
\text { o descongelamento, \% }\end{array}$ & $51,7 \pm 1,86 \mathrm{c}$ & $68,7 \pm 1,67 \mathrm{a}$ & $59,5 \pm 3,27 b$ & $63,2 \pm 3,59 b$ & $68,3 \pm 1,58 \mathrm{a}$ & $72,2 \pm 2,39 a$ \\
\hline $\begin{array}{l}\text { Integridade de } \\
\text { membrana plasmática } \\
\text { baseada em } \\
\text { fluorescência após o } \\
\text { descongelamento, \% }\end{array}$ & $34,2 \pm 2,28 \mathrm{a}$ & $4,6 \pm 0,62 \mathrm{c}$ & $25,7 \pm 2,41 b$ & $6,1 \pm 0,80 \mathrm{c}$ & $30,9 \pm 1,32 \mathrm{a}$ & $21,4 \pm 2,58 b$ \\
\hline $\begin{array}{l}\text { Integridade de } \\
\text { membrana após o } \\
\text { descongelamento na } \\
\text { avaliação HOST, \% }\end{array}$ & $19,0 \pm 1,26 \mathrm{a}$ & $13,6 \pm 1,30 \mathrm{~b}$ & $18,1 \pm 1,57 \mathrm{a}$ & $9,8 \pm 0,78 \mathrm{c}$ & $20,6 \pm 1,07 \mathrm{a}$ & $20,7 \pm 1,25 \mathrm{a}$ \\
\hline
\end{tabular}

$\mathrm{Na}$ avaliação do percentual de espermatozoides com morfologia normal após o descongelamento, os tratamentos com a inclusão do etilenoglicol EG-GEMA e EG-LDL, além do tratamento DMF-GEMA, não diferiram entre si $(\mathrm{P}>0,05)$, mas foram melhores que os demais $(\mathrm{P}<0,05)$. A combinação GLI-GEMA foi a de pior resultado relacionado à morfologia normal 51,7 $\pm 1,86$. 
Os tratamentos GLI-GEMA e EG-GEMA foram os que proporcionaram os melhores resultados de membranas plasmáticas íntegras no descongelamento avaliadas por meio de sondas fluorescentes, sendo superiores aos demais $(\mathrm{P}<0,05)$. Os tratamentos GLI-LDL e EG-LDL não diferiram entre si $(\mathrm{P}>0,05)$. Semelhante ao observado na avaliação da motilidade após o descongelamento, os piores resultados de integridade de membrana plasmática ocorreram nos tratamentos DMF-GEMA e DMF-LDL.

A avaliação da integridade de membrana espermática por meio do HOST mostrou não haver diferença $(\mathrm{P}>0,05)$ entre os tratamentos que incluíram glicerol GLI-GEMA e GLI-LDL em relação àqueles que incluíram EG-GEMA e EG-LDL, enquanto o percentual de membranas íntegras no HOST para os tratamentos DMFGEMA e DMF-LDL, além de diferirem entre si $(\mathrm{P}<0,05)$, também foram os resultados mais baixos $(\mathrm{P}<0,05)$.

\section{DISCUSSÃO}

O maior percentual de motilidade espermática após o descongelamento foi obtido na combinação GLI-GEMA, porém esse tratamento apresentou alto percentual de patologia espermática, especialmente quando comparado aos tratamentos que utilizaram etilenoglicol e com a combinação DMF-GEMA. Sabidamente o glicerol pode causar danos à morfologia espermática (Watson, 2000). Assim, considerando as recomendações do CBRA (Manual, 1998) e a combinação das avaliações de motilidade e morfologia, os tratamentos EGGEMA e EG-LDL estariam de acordo com recomendação brasileira, pois teriam motilidade acima de $30 \%$ e, no máximo, $30 \%$ de patologias. No entanto, quando a integridade da membrana plasmática dos espermatozoides foi considerada, as combinações que resultaram em melhores resultados foram GLI-GEMA e EG-GEMA, apresentando uma relação interessante com a avaliação de motilidade, já demonstrado por Bianchi et al. (2008).

A criopreservação do sêmen de qualquer espécie implica perda da qualidade seminal (Watson, 2000; Yoshida, 2000) em razão dos danos provocados nas membranas celulares (Maxwell e Johnson, 1997). A integridade estrutural e funcional das membranas é, portanto, fundamental para a viabilidade do espermatozoide. O glicerol e a gema de ovo são amplamente utilizados nos protocolos de congelamento de sêmen de touro (De Leeuw et al., 1993; Ollero et al., 1998; Wall and Foote, 1999; Moussa et al., 2002; Amirat et al., 2004). A ação crioprotetora da gema de ovo é atribuída à fração LDL, em que vários estudos têm demonstrado que a LDL pode aumentar a resistência espermática contra o choque térmico, melhorar a motilidade, a integridade do acrossoma e da membrana plasmática (Graham e Foote, 1987; Demaniowicz e Strzezek, 1996; Moussa et al., 2002). Trabalhos com LDL na composição de diluentes para sêmen foram realizados em diferentes espécies. A substituição da gema de ovo por LDL foi benéfica para a manutenção da qualidade espermática em sêmen de cães quando preservado à temperatura de refrigeração a $5^{\circ} \mathrm{C}$ ou congelado (Varela Junior et al., 2009), bem como de sêmen refrigerado (Demaniowicz e Strzezek, 1996) e sêmen congelado de suínos (Hu et al., 2006).

O uso da LDL em substituição à gema não contribuiu para melhora dos parâmetros de qualidade espermática ao descongelamento. As implicações da relação dos crioprotetores com a complexidade estrutural da membrana espermática, bem como a etapa durante o processo de congelamento que mais altera a dinâmica estrutural da membrana, e como os componentes crioprotetores interagem com esta, ainda não estão bem esclarecidos, o que dificulta a adoção de medidas com o objetivo de melhor proteger a membrana durante o processo de criopreservação (Parks e Graham, 1992). Dessa forma, o percentual de inclusão de LDL neste trabalho talvez tenha sido insuficiente para promover os benefícios que foram obtidos em outros trabalhos (Manjunath et al., 2002; Anton et al., 2003; Bergeron et al., 2004).

Desde a descoberta por Polge et al. (1949) do efeito crioprotetor do glicerol, ele tornou-se o crioprotetor intracelular mais utilizado na criopreservação de sêmen bovino. A tentativa, neste trabalho, de substituição do glicerol por dimetilformamida não foi efetiva, tendo em vista os baixos resultados obtidos na avaliação de motilidade e integridade de membrana do sêmen descongelado, tanto no tratamento DMF-GEMA como DMF-LDL. A menor viscosidade e maior permeabilidade de membrana das amidas (Ball e 
Vo, 2001) pode ter ocasionado maior estresse osmótico na célula espermática, prejudicando a qualidade seminal ao descongelamento. Já o uso do etilenoglicol combinado especialmente com a gema de ovo (EG-GEMA) parece ser uma alternativa interessante ao uso de GLI-GEMA, pois possibilitou os maiores percentuais de espermatozoides com morfologia normal e integridade de membrana plasmática dos espermatozoides, tanto na avaliação por fluorescência como pelo HOST.

\section{CONCLUSÃO}

O uso da combinação EG-GEMA pode ser utilizada com eficiência na criopreservação de sêmen de touros, enquanto que o uso de dimetilformamida e LDL não promoveu melhora na qualidade seminal.

\section{REFERÊNCIAS}

ALVARENGA, M.A.; LANDIM-ALVARENGA, F.C.; MOREIRA, R.M. et al. Acrossomal ultrastructure of stallion spermatozoa cryopreserved with ethylene glycol using two packaging systems. Equine Vet. J., v.32, p.541$545,2000$.

AMIRAT, L.; TAINTURIER, D.; JEANNEAU, L. et al. Bull semen in vitro fertility after cryopreservation using egg yolk LDL: a comparison with Optidyl $^{\circledR}$, a commercial egg yolk extender. Theriogenology, v.61, p.895-907, 2004.

ANTON, M. Chemical and structural characterization of low-density lipoproteins purified from hen egg yolk. Food Chem., v.83, p.175-183, 2003.

BALL, B.A.; VO, A. Osmotic tolerance of equine spermatozoa and the effects of soluble cryoprotectant on equine sperm motility, viability and mitochondrial membrane potential. J. Androl., v.22, p.1061-1069, 2001.

BEARDEN, H.J.; FUQUAY, J.W. Semen evaluation. In: Bearden, H.J.; Fuquay, J.W. (Ed.). Applied Animal Reproduction. $4^{\text {th }}$ Ed. New Jersey: Prentice Hall. Cap. 15, 1997. p.159-170.
BERGERON, A.; CRÊTE, M.H.; BRINDLE, Y.; MANJUNATH, P. Low-density lipoprotein fraction from hen's egg yolk decreases the binding of the major protein of bovine seminal plasma to sperm and prevents lipid efflux from the sperm membrane. Biol. Reprod., v.70, p.708717, 2004.

BERGERON, A.; MANJUNATH, P. New insights towards understanding the mechanisms of sperm protection by egg yolk and milk. Molec. Reprod. Develop., v.73, p.1338-1344, 2006.

BIANCHI, I.; CALDERAM, K.; MASCHIO, E.F. et al. Evaluation of amides and centrifugation temperature in boar semen cryopreservation. Theriogenology, v.69, p.632638, 2008.

COLÉGIO BRASILEIRO DE REPRODUÇÃO ANIMAL. CBRA. 1998. Manual para exame andrológico e avaliação de sêmen animal. Colégio Brasileiro de Reprodução Animal. 2.Ed. Belo Horizonte: CBRA, p.49.

DE LEEUW, F.E.; DE LEEUW, A.M.; DEN DAAS, J.H. et al. Effects of various cryoprotective agents and membrane-stabilizing compounds on Bull sperm membrane integrity after cooling and freezing. Cryobiology, v.30, p.32-44, 1993.

DEMANIOWICZ, W.; STRZEZEK, J. The effect of lipoprotein fraction of egg yolk on some of the biological properties of boar spermatozoa during storage of the semen in liquid state. Reprod. Dom. Anim., v.31, p.279-280, 1996.

GRAHAM, J.K.; FOOTE, R.H. Effect of several lipids fatty acyl chain length and degree of unsaturation on the motility of bull spermatozoa after cold shock and freezing. Cryobiology., v.24, p.42-52, 1987.

GUTHRIE, H.D.; LIU, J.; CRITSER, J. Osmotic tolerance limits and effects of cryoprotectants on motility of bovine spermatozoa. Biol. Reprod., v.67, p.1806-1811, 2002.

HARRISON, R.A.P.; VICKERS, S.E. Use of fluorescent probes to assess membrane integrity in mammalian spermatozoa. J. Reprod. Fert., v.88, p.343-352, 1990. 
HU, J.H.; LI, Q.W.; LI, G. et al. The cryoprotective effect on frozen-thawed boar semen of egg yolk low density lipoproteins. Asian-Australasian J. Anim. Sci., v.19, p.486490, 2006.

JIANG, Z.L.; LI, Q.W.; HU, J.H. et al. Improvement of the quality of boar cryopreservation semen by supplementing with low density lipoprotein in diluents. Cryobiology, v.54, p.301-304, 2007.

MANJUNATH, P.; NAUC, V.; BERGERON, A.; MÉNARD, M. Major Proteins of bovine seminal plasma bind to the low-density lipoprotein fraction of hen's egg yolk. Biol. Reprod., v.67, p.1250-1258, 2002.

MANJUNATH, P.; THÉRIEN, I. Role of seminal plasma phospholipids binding proteins. J. Reprod. Immuno., v.53, p.109-119, 2002.

MAXWELL, W.M.C.; JOHNSON, L.A. Membrane status of boar spermatozoa after cooling or cryopreservation. Theriogenology, v.48, p.209-219, 1997.

MEDEIROS, A.S.L.; FORELL, F.; OLIVEIRA, A.T.D.; RODRIGUES, J.L. Cryopreservation of stallion sperm using different amides. Theriogenology, v.58, p.273-276, 2002.

MOUSSA, M.; MARTINET, V.; TRIMECHE, A. et al. Low density lipoproteins extracted from hen egg yolk by an easy method: cryoprotective effect on frozen-thawed bull semen. Theriogenology, v.57, p.1695-1706, 2002.

NAGASE, H.; TOMIZUCA, T; HANADA, A. et al. Crioprotective action of some amides on espermatozoa of domestical animal. Efects of formamide, acetamide and lactamide on the motility of bovine spermatozoa after pellets freezing. Japan. J. Anim. Reprod., v.18, p.15-21, 1972.

OGIER DE BAULNY, B.; LABBÉ, C.; MAISSE, G. Membrane integrity, mitochondrial activity, ATP content and motility of European Catfish (Silurus glanis) testicular spermatozoa after freezing with different cryoprotectants. Cryobiology., v.39, p.177-184, 1999.

OLLERO, M.; BESCÓS, O.; CEBRIÁNPÉREZ, J.A. et al. Loss of plasma membrane proteins of bull spermatozoa through the freezing-thawing process. Theriogenology, v.49, p.547-555, 1998 .
PARKS, J.E.; GRAHAM, J.K. Effects of cryopreservation procedures on sperm membranes. Theriogenology, v.38, p.209-223, 1992.

POLGE, C.; SMITH, A.U.; PARKES, A.S. Revival of spermatozoa after vitrification and dehydration at low temperatures. Nature, v.164, p.666, 1949.

SHIN, S.J. et al. A new antibiotic combination for frozen bovine semen. Theriogenology, v.29, p.577-591, 1988.

SQUIRES, E.L.; KEITH, S.L.; GRAHAM, J.K. Evaluation of alternative cryoprotectants for preserving stallion spermatozoa. Theriogenology, v.62, p.1056-1065, 2004.

STATISTICAL Analisys Sistem - SAS®. SAS/STAT User's Guide (Release 6.03). SAS Inst. Inc., Cary, NC, 1999.

TSELUTIN, K.; SEIGNEURIN, F.; BLESBOIS, E. Comparison of cryoprotectants and methods of cryopreservation of fowl spermatozoa. Poult. Sci., v.78, p.586-590, 1999.

VARELA, JUNIOR. A.S.; CORCINI, C.D.; ULGUIM, R.R., et al. Effect of low density lipoprotein on the quality of cryopreserved dog semen. Anim. Reprod. Sci., v.115, p.323-327, 2009.

VASQUEZ, J.M.; MARTINEZ, E.A.; MARTINEZ, P. et al. Hypoosmotic swelling of boar spermatozoa compared to other methods for analysing the sperm membrane. Theriogenology, v.47, p.913922, 1997.

WALL, R.J.; FOOTE, R.H. Fertility of bull sperm frozen and stored in clarified egg yolktris-glycerol extender. J. D. Sci., v.82, p.817-821, 1999.

WATSON, P.F. The causes of reduced fertility with cryopreserved semen. Anim. Reprod. Sci., v.60-61, p.481-492, 2000

YOSHIDA, M. Conservation of sperms: current status and new trends. Anim. Reprod. Sci., v.6061, p.349-355, 2000. 